\title{
Dinámica del mercado de arroz en san Martín y Lambayeque, Perú: relación con compras públicas durante el período 2006-2011
}

\author{
Dynamics of the rice market in San Martin and Lambayeque, peru: relationship with public \\ purchases during 2006-2011
}

\author{
Karina Maldonado C. ${ }^{1}$; Jorge A. Alarcón N.
}

\begin{abstract}
Resumen
En este artículo se analizan los procesos de compra realizados por entidades estatales de las regiones Lambayeque y San Martín, en Perú (2006-2011), con el propósito de evaluar los efectos de la presencia del Estado, como demandante de arroz, en el mercado regional. Se realizó una clasificación de los proveedores estatales y se estimaron modelos multivariados para explicar el comportamiento y los determinantes de la producción y los precios (en chacra) de arroz, en ambas regiones. Las estadísticas presentadas muestran, básicamente, que la magnitud de las compras públicas de arroz, respecto al mercado privado, son muy pequeñas para inducir efectos importantes sobre la producción total de arroz en las regiones estudiadas. Además, las compras públicas tuvieron un comportamiento tal que no siempre se concentraron en los periodos de mayor producción de arroz. En cambio sí se pudo constatar un efecto importante de precios pagados por el Estado, con respecto a precios del arroz en chacra, lo que se nota con mayor énfasis cuanto mayores son los precios relativos pagados por las entidades estatales; una sugerente explicación de este último resultado es la sensibilidad histórica de los precios en chacra respecto a la tendencia de altos precios pagados por el Estado.
\end{abstract}

Palabras clave: compras públicas; productores agrícolas; producción y precios de arroz; Perú.

\begin{abstract}
In this paper we analyzed the purchasing processes conducted by state entities of the Lambayeque and San Martin regions in Peru (2006-2011), with the ultimate purpose of evaluating the effects of State presence as rice buyer in the regional market. A classification of state suppliers was made and multivariate models were estimated to explain the behavior and determinants of rice production and prices of rice (on the farm), in both regions. The statistics presented show basically that the magnitude of rice purchases by public entities, compared to the private rice market, in both regions, is too small to induce significant effects on regional rice production. In addition, government purchases of rice behaved differently to the production, and did not always concentrate on periods of higher rice production. On the other hand, it was possible to observe a significant effect of prices paid by the state, with respect to farm-gate rice prices, which is more noticeable the grater the relative prices paid by the state entities. A suggestive explanation of the latter result is the historical sensitivity of farm prices in relation to the tendency of high prices paid by the state.
\end{abstract}

Keywords: public procurement; agricultural producers; production and prices of rice; Perú.

\section{Introducción}

El mercado de las compras públicas es un gran espacio para que el Gobierno peruano ejecute políticas públicas mediante incentivos a algunos sectores productivos. Es el caso, por ejemplo, de la normativa que ha previsto brindar un trato especial a las micro y pequeñas empresas (MYPES), que gozan de preferencias cuando la entidades estatales requieren contratar bienes y servicios ofertados por estas.
El sector agropecuario no es ajeno a este beneficio ya que la norma peruana permite a las entidades públicas encargadas de los programas sociales, como fue el caso del Programa Nacional de Asistencia Alimentaria (PRONAA), de los Programas de Compensación Social y de otros organismos del Estado, adquirir directamente productos alimenticios a pequeños productores locales, a fin de incentivar esta producción y reducir la brecha de los productos agropecuarios, con relación a la producción proveniente de otros sectores.

1 Economista. Universidad Nacional Agraria La Molina, Lima, Perú. E-mail: karinamaldonadoc@gmail.com

2 Profesor Principal. Universidad Nacional Agraria La Molina, Lima, Perú. E-mail: alarcon1956@gmail.com 
En el Perú, el arroz es un producto importante entre los adquiridos por los programas sociales del Gobierno, ya que es un alimento básico del habitante peruano y cubre alrededor del $19 \%$ del total diario de calorías requeridas. El consumo de arroz en el Perú, además, ha evolucionado significativamente, pasando de 25 a $54 \mathrm{~kg}$ per cápita al año, en los últimos 20 años, llegando a ser el más alto de Latinoamérica (FAO, 2009).

Debido a que el arroz es un producto demandado por toda la población, las compras estatales se ven minimizadas en comparación con las compras realizadas por el sector privado. Por otro lado, las compras de arroz realizadas por el Estado, por norma, deberían enfocarse en los productores locales y no en los mayoristas y minoristas, motivo por el cual ha sido necesario realizar un análisis departamental en las principales regiones productoras (hacemos la salvedad que en este documento se usa el término compras estatales como equivalente de "compras del sector público" o compras de entidades públicas).

La producción de arroz está centralizada en el nororiente peruano, siendo las regiones más importantes: Lambayeque, Piura y San Martín, que concentran el 50 $\%$ del total producido en el país. En el caso de Piura, los productores venden el arroz en cáscara, principalmente, con lo que no atienden directamente la demanda estatal. En contraste, en Lambayeque y San Martín los productores han avanzado en la cadena de valor, vendiendo predominantemente arroz pilado. Sin embargo, existen también diferencias importantes entre estas dos últimas regiones del país (Gonzales et al., 2010).

El problema es que los pequeños productores de arroz tienen pocos mecanismos de venta óptima de su producto. Por su parte, las entidades públicas han sido y son compradoras importantes de arroz, aunque no se conoce precisamente si su actividad demandante ha tenido algún efecto significativo y equitativo en la producción y precios de arroz provenientes de las principales zonas productoras del país. Este conocimiento es importante para la determinación y aplicación de políticas públicas en el país.

El objetivo general es evaluar el efecto que tienen las compras públicas de arroz en la dinámica del mercado regional de este producto, en los departamentos de San Martín y Lambayeque (2006-2011). Para conseguir este objetivo, se analizarán las características de la provisión de arroz de las entidades públicas demandantes de las regiones San Martín y Lambayeque y se establecerá la magnitud del efecto de cantidades y precios ofertados por entidades públicas, con relación a volúmenes y precios de arroz en el mercado de las regiones en estudio. En la sección de Materiales y Métodos se incluye una breve revisión de la literatura que permite entender mejor el contexto del trabajo.

\section{Materiales y métodos}

\section{Revisión de Literatura}

Las compras públicas son una herramienta importante con la que cuentan los gobiernos para realizar políticas de incentivos a algunos sectores productivos. Por el lado de la oferta, es un espacio para que los productores puedan ofrecer bienes y servicios de calidad a uno de los principales demandantes, que suele ser el Estado. En tal medida, este último puede dirigir su demanda con el objetivo de dinamizar el mercado interno.

Un aspecto importante del desarrollo de las adquisiciones de las entidades públicas son las compras estatales "discriminatorias". Este tipo de compra se presenta cuando la demanda pública está orientada a la adquisición directa de bienes y servicios producidos o prestados en el propio país (es decir, "discrimina" la producción extranjera). Tal acción podría dar como resultado un incremento de la producción local y la reducción de importaciones en ciertos sectores de la economía. La característica clave de la cual dependen los resultados de estas prácticas, es el tamaño del Estado como demandante "discriminatorio" dentro del mercado (Trionfetti, 2000). Un aspecto crítico podría ser que el Estado tenga que pagar precios más altos y enfrentar opciones limitadas para elegir los proveedores, lo que comprometería el interés público.

En la región latinoamericana, las pequeñas y medianas empresas (PYMES) han sido actores importantes en el desarrollo productivo de los países de la región, especialmente en lo que se refiere a la generación de empleo. Asimismo, se ha estimado que las pequeñas y medianas empresas actualmente participan con un 20 al $30 \%$ de las compras y contrataciones públicas totales. Destaca particularmente el caso de Chile con más del 50 $\%$ de participación, mientras que Perú, Ecuador, Paraguay y Uruguay se ubican con una participación de alrededor del $50 \%$ (Beláustegui, 2011).

Respecto a los resultados de la relación entre compras públicas y producción agraria en la región latinoamericana, podría decirse que el desarrollo de programas sociales, ocasionalmente, ha mostrado tener un impacto positivo a corto plazo en la pobreza rural (Gómez y Nieto, 2006; FAO, 2013). En Perú, la política general de contrataciones del Estado peruano está en función de lo que establece la Ley de Contrataciones del Estado, reglamentada por el Ministerio de Economía y Finanzas, en coordinación con el Organismo Supervisor de las Contrataciones del Estado (OSCE). La relación con el agro se ha dado, fundamentalmente, mediante prácticas consideradas “discriminatorias". Durante 1992 y 2012, la entidad demandante más importante fue el PRONAA, institución cuyo objetivo fue prevenir la malnutrición en sectores de pobreza y pobreza extrema, pero actualmente desaparecida.

En el caso particular de los alimentos, tres han sido los principales instrumentos que conforman el marco legal peruano: las Leyes 26850 y 27060 , así como los dispositivos relativos al subsidio de comedores (hasta el 2012 la Ley 27060 establecía la adquisición directa de productos alimenticios a productores locales). En los últimos años, el monto de inversión pública en compras del 
Estado peruano ha ascendido a cifras anuales próximas a los US\$ 10.000 millones, al cual acceden los proveedores locales, gracias a las medidas reseñadas anteriormente. Por otro lado, las adquisiciones en el marco de la Ley 27060 (compra directa), han representado entre el $69 \%$ y el $89 \%$, aproximadamente, del total de las adquisiciones de productos agropecuarios (Rojas y Effio, 2012). Hasta el 2012, época del PRONAA, el producto autorizado, adjudicado y programado en mayor cantidad fue el arroz corriente mejorado. Eventualmente, algunos estudios en el Perú han sustentado efectos positivos de las compras públicas en el agro peruano (Rojas y Flores, 2009; Rebosio y Rodríguez, 2001); pero, a menudo, el rol del PRONAA fue calificado como meramente "asistencialista"; no habiéndose sustentado finalmente efectos positivos en el mercado de los productores agrarios en general.

\section{Modelos econométricos}

Este trabajo combina el uso de estadística descriptiva con un modelo de regresión multivariado que utiliza series de tiempo para establecer el grado de relación entre compras públicas y producción/precios de arroz para el período 2006-2011.

La estadística descriptiva ha sido utilizada para dar una visión integral del "comportamiento" de las compras de arroz realizadas por el Estado peruano, además de determinar su importancia en el mercado de dicho cultivo en Lambayeque y San Martín. Mediante estadística descriptiva, se identificó a los proveedores de ambas regiones, los que fueron analizados y caracterizados de acuerdo al tipo de comercialización de arroz. Además, se determinó la participación que poseen los productores dentro del mercado estatal de arroz, es decir, la porción de la demanda estatal que es atendida por los productores agrícolas y las asociaciones de productores.

El modelo de regresión multivariado general tuvo la siguiente forma funcional:

$Y_{t}=\beta_{0}+\beta_{1}\left(X_{1 t}\right)+\ldots+\beta_{k}\left(X_{k t}\right)+\mu_{t}$

Donde:

$Y$ : Variable respuesta.

$\boldsymbol{\beta}_{0}$ : Es el intercepto, indica el nivel de $\boldsymbol{Y}$ cuando las variables regresoras $(\mathbf{X})$ son cero.

$\boldsymbol{\beta}_{1} \ldots \boldsymbol{\beta}_{\mathrm{k}}$ : Son coeficientes de regresión parcial y nos indican el cambio en el valor de $\boldsymbol{Y}$ por unidad de cambio en cada una de las regresoras $(\boldsymbol{X})$.

$\boldsymbol{\mu}_{\mathbf{t}}$ : Es el término de error.

\section{Identificación de variables}

Para cada una de las regiones señaladas se utilizaron dos modelos (con la especificación indicada líneas arriba), con variables consignadas en las Tablas 1 y 2 .

\section{Validación estadística del modelo}

La validación del modelo incluye el manejo de lo siguiente: (1) Especificación, que consistió en verificar si las variables explicativas son relevantes o significativas

Tabla 1. Variables del modelo econométrico que explica el comportamiento de la producción de arroz en cada región

\begin{tabular}{|l|l|}
\hline $\begin{array}{l}\text { VARIABLE } \\
\text { DEPENDIENTE }\end{array}$ & VARIABLES INDEPENDIENTES \\
\hline \multirow{4}{*}{$\begin{array}{l}\text { Producción de } \\
\text { arroz pilado en } \\
\text { toneladas por } \\
\text { mes }^{(1)}\end{array}$} & Demanda estatal: cantidad (en toneladas de arroz pilado) mensual que adquirió el Estado \\
\cline { 2 - 2 } & Precio promedio mensual en chacra de arroz cáscara en nuevos soles por tonelada \\
\cline { 2 - 2 } & Precio promedio mensual del arroz en la ciudad principal de la región, en nuevos soles por Tm \\
\cline { 2 - 2 } & Precio promedio mensual pagado por el Estado, en nuevos soles por tonelada de arroz pilado \\
\cline { 2 - 2 } & Número de proveedores que atendieron la demanda estatal por mes ${ }^{(2)}$ \\
\cline { 2 - 2 } & Área cosechada de arroz en hectáreas \\
\hline
\end{tabular}

Fuentes: ${ }^{(1)}$ Reportes mensuales agrarios, WEB-MINAG ${ }^{(2)}$ Base de datos WEB-SEACE.

Tabla 2. Variables del modelo econométrico que explica el comportamiento del precio en chacra de arroz en cada región

\begin{tabular}{|c|c|}
\hline $\begin{array}{l}\text { VARIABLE } \\
\text { DEPENDIENTE }\end{array}$ & VARIABLES INDEPENDIENTES \\
\hline \multirow{6}{*}{$\begin{array}{l}\text { Precio promedio } \\
\text { mensual en chacra } \\
\text { de arroz cáscara, } \\
\text { en nuevos soles } \\
\text { por tonelada }^{(1)}\end{array}$} & Producción de arroz pilado en toneladas por mes ${ }^{(1)}$ \\
\hline & Demanda estatal: cantidad (en toneladas de arroz pilado) mensual que adquirió el Estado ${ }^{(2)}$ \\
\hline & Precio promedio mensual del arroz en la ciudad principal de la región, en nuevos soles por $\mathrm{Tm}^{(1)}$ \\
\hline & Precio promedio mensual pagado por el Estado, en nuevos soles por tonelada de arroz pilado ${ }^{(2)}$ \\
\hline & Número de proveedores que atendieron la demanda estatal por mes ${ }^{(2)}$ \\
\hline & Área cosechada de arroz en hectáreas ${ }^{(1)}$ \\
\hline
\end{tabular}

Fuentes: ${ }^{(1)}$ Reportes mensuales agrarios, WEB- MINAG $\left.{ }^{(2}\right)$ Base de datos WEB SEACE. 
y si son las que más contribuyen a la explicación del comportamiento de la variable dependiente; (2) La linealidad, que implica particularmente la forma cómo se relacionan las variables regresoras con la variable dependiente (expresada por los parámetros); (3) La evaluación y arreglo del problema de multicolinealidad (grado "importante" de asociación entre las variables explicativas), que fue detectado con correlaciones simples y factores de inflación de varianzas; corregido con eliminación de variables menos relevantes; (4) Uso de errores estándar robustos Newey-West, que permitió el arreglo de perturbaciones no esféricas (heteroscedasticidad y autocorrelación).

Para ambos modelos también se incluyó una variable de "tendencia", a efectos de aislar tal efecto (tendencia), respeto a la relación "genuina" entre variables regresoras y variable objetivo. Además, dada la naturaleza temporal de la base de datos, en el caso de la región Lambayeque se incluyó una variable ficticia (dummy o dicotómica) que toma el valor de "0" para los meses de producción alta y " 1 " en los demás meses. Esta variable fue utilizada con el propósito de "desestacionalizar" el modelo y conseguir mejores estimadores de los parámetros de regresión (Wooldridge, 2009).

\section{Fuentes y arreglo de datos}

Se analizaron una serie de variables determinantes de la producción y precios de arroz en Lambayeque y San Martín. La base de datos para la alimentación de los modelos de las Tablas 1 y 2 corresponden a series de tiempo mensual, con período de análisis de seis años (2006-2011), lo que hace un total de 41 observaciones. Las fuentes de información secundaria han sido: bases de datos del (1) el Ministerio de Agricultura - MINAG y (2) Organismo Supervisor de Contrataciones del Estado - OSCE.

\section{Resultados y discusión}

Los resultados aquí reportados se basan en estadísticas formales. Los procesos informales, eventualmente comentados, en relación a la participación de los productores, no son comprendidos en este estudio.

\section{Tipología de Proveedores Estatales de Arroz \\ Clasificación de proveedores}

Según el Registro Nacional de Proveedores (RNP), la clasificación de proveedores es respecto al tipo de bien o servicio que proveen existiendo las siguientes opciones: (i) Proveedor de Bienes, (ii) Proveedor de Servicios, (iii) Ejecutor de Obras y (iv) Consultor de Obras. Sin embargo, para el caso de los proveedores de arroz, se necesitó hacer una caracterización de la principal actividad económica de tales proveedores, de manera que se pudo mostrar la participación de los productores agrícolas y sus asociaciones.

Por la razón anterior, la caracterización se realizó de acuerdo al tipo de contribuyente y a la actividad principal que realizan según la base de datos de la Superintendencia
Nacional de Administración Tributaria (SUNAT), considerándose los siguientes tipos de proveedores de arroz: (i) Productor Agrario (Persona natural, cuya actividad principal es el cultivo de cereales),(ii)Asociación de Productores Agrarios (Persona jurídica, integrada por productores agrícolas), (iii) Comerciante de Productos Agrícolas (Persona natural, cuya actividad principal es la comercialización de alimentos de procedencia agrícola), (iv) Empresa Comercializadora de Productos Agrícolas (Empresa dedicada a la comercialización de alimentos del agro), (v) Empresa Agroindustrial (empresa cuya actividad principal es la producción, procesamiento y/o el comercio de alimentos de procedencia agrícola).

\section{Procesos de selección y entidades demandantes}

Cuando se trata de adquisiciones dirigidas a beneficiar a los productores locales, estos deben contar con ciertos requisitos y condiciones (procesos descritos en la web del SEACE-Perú). En los procesos de selección procede la calificación técnica y también la económica.

En los procesos clásicos de selección, normalmente se fija un máximo en la cantidad ofertada por postor, a fin de adjudicar el proceso a prorrata (repartición proporcional entre varios, beneficiando un mayor número de proveedores. Igual sucede en algunos casos donde el límite máximo de propiedad de tierras determina la participación en los procesos de selección. Ambas situaciones dependen de la cantidad de arroz que se considera en el proceso de adquisición. Una modalidad distinta, también usada, es la de "Subasta Inversa", por la cual las entidades públicas adquieren bienes a través de un procedimiento muy rápido, en el cual solamente se califica el precio que ofrecen los participantes. El "comprador" expresa o presenta el bien que necesita con detalles y especificaciones; los "vendedores" ofrecen sus productos y precios. El vendedor que ofrece el precio más bajo 'gana' la subasta (bajo esta última modalidad, prácticamente quedan "fuera del mercado" aquellos proveedores (o productores) que no cuenten con la capacidad de reducir sus costos y ofrecer precios más bajos.

En la Fig. 1 se muestra la cantidad de procesos de adquisición de arroz de acuerdo a la modalidad y tipo de proceso. Se puede observar que en la región Lambayeque la mayor parte de los procesos que se realizaron, durante el periodo de análisis, estuvieron bajo la modalidad de subasta inversa, favoreciendo la presencia de intermediarios con capacidad de acopiar grandes cantidades y ofrecer menores precios. Sucede lo contrario en la región San Martin, donde la frecuencia de procedimiento bajo la modalidad de subasta inversa, es significativamente menor respecto a lo realizado bajo el procedimiento clásico. Lo anterior podría explicar porque la participación de los productores locales, especialmente en asociaciones, es mucho mayor en esta región.

Participación de productores locales en el mercado estatal de arroz

Durante el periodo de análisis (2006-2011), en 


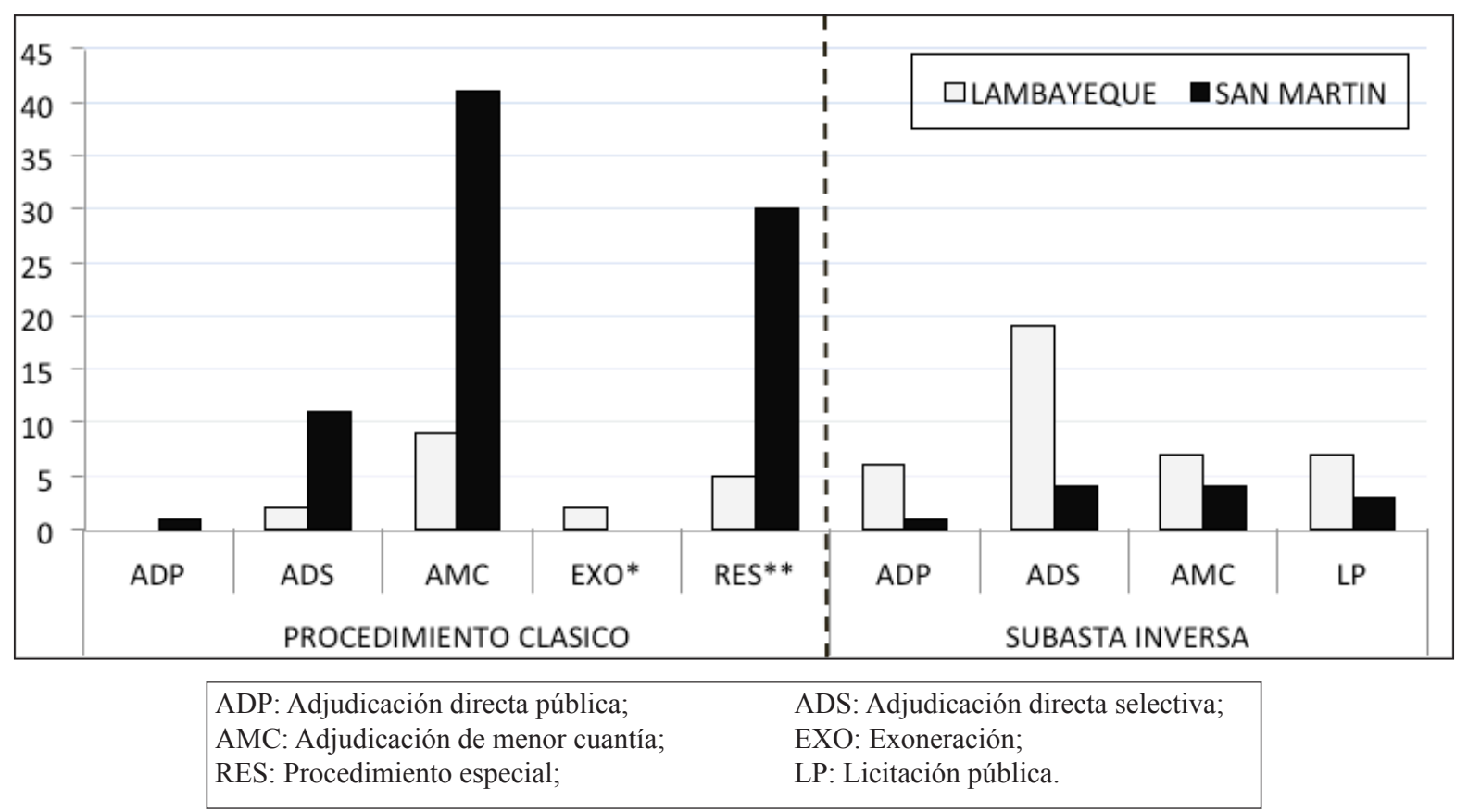

Figura 1. Perú: $\mathrm{N}^{\circ}$ de procesos, por tipo de proceso y región (2006 - 2011)

Fuente (SEACE).

${ }^{(*)}$ Exoneraciones de procesos de selección, Art. $20^{\circ}$ de la LCE. ${ }^{(* *)}$ Incluye los procesos de selección realizados bajo las siguientes normas Ley $\mathrm{N}^{\circ} 27060$ y Ley $\mathrm{N}^{\circ} 27767$.

Lambayeque y San Martín, fueron 348 proveedores los que atendieron la demanda pública de casi 95 millones de nuevos soles. En la Tabla 3 puede notarse que la cantidad total de proveedores en San Martín fue más del doble de la cantidad de proveedores en la región Lambayeque. Respecto al monto adjudicado, las cantidades son aun significativamente mayores en San Martín: S/. 81,13 millones versus S/. 13,74 millones en Lambayeque. Asimismo, se puede observar que en la región Lambayeque son los productores y las asociaciones de productores quienes tienen una mayor participación, con el 53,3\% y el 19,0\% respectivamente; sin embargo, la participación de las empresas comercializadoras de arroz es, también, considerable alcanzando la cifra del 16,2\%. Por otro lado, en San Martín se nota que las asociaciones de productores representan la mayor parte del total de proveedores estatales de arroz en la región (alcanzando una cifra alrededor del $86 \%$ ). Además, la presencia de las personas y empresas dedicadas al comercio de arroz tienen una participación mínima; sin embargo son superiores a la participación de los productores que contrataron con el Estado en forma individual, que es solo del 2,5\%.

Efecto de compras y precios estatales en la producción Los modelos de regresión, en esta sección, pretenden explicar la relación de causalidad existente entre un conjunto de variables determinantes y la producción de arroz (toneladas mensuales), para las regiones Lambayeque y San Martín, de manera separada. Después de varias especificaciones funcionales (entre las que destacan la lineal, semi-logarítmica y doble-logarítmica), así como el uso de algunos indicadores de la bondad de ajuste (coeficientes de determinación, error estándar de

Tabla 3. Perú: Número de proveedores estatales, según tipo y región (2006 - 2011)

\begin{tabular}{|l|c|c|c|c|}
\hline & \multicolumn{2}{|c|}{ LAMBAYEQUE } & \multicolumn{2}{c|}{ SAN MARTÍN } \\
\hline \multirow{2}{*}{ TIPO DE PROVEEDOR } & \multirow{2}{*}{ N $^{\circ}$ DE PROVEEDORES } & $\%$ & \multirow{2}{*}{ N $^{\circ}$ DE PROVEEDORES } & $\%$ \\
\hline Productor & 56 & $53,3 \%$ & 6 & $2,5 \%$ \\
\hline Asociación & 20 & $19,0 \%$ & 210 & $86,1 \%$ \\
\hline Comerciante & 10 & $9,5 \%$ & 9 & $3,7 \%$ \\
\hline Empresa comercializadora & 17 & $16,2 \%$ & 13 & $5,3 \%$ \\
\hline Empresa agroindustrial & -- & -- & 1 & $0,4 \%$ \\
\hline Otros & 2 & $1,9 \%$ & 5 & $2,0 \%$ \\
\hline \multicolumn{1}{|c|}{ TOTAL } & $\mathbf{1 0 5}$ & $\mathbf{1 0 0 , 0} \%$ & $\mathbf{2 4 4}$ & $\mathbf{1 0 0 , 0 \%}$ \\
\hline
\end{tabular}

Fuente: Webs de SEACE y SUNAT- Perú. 
la regresión, prueba $F$ múltiple y los criterios Akaike y Schwarz), se determinó que la ecuación funcional que mejor representó el ajuste a los datos disponibles fue la función lineal:

$\mathrm{Y}_{\mathrm{t}}=\boldsymbol{\beta}_{0}+\boldsymbol{\beta}_{1}\left(\mathrm{X}_{1 \mathrm{t}}\right)+\ldots+\boldsymbol{\beta}_{\mathrm{k}}\left(\mathrm{X}_{\mathrm{kt}}\right)+\boldsymbol{\mu}_{\mathrm{t}}$

Donde $\boldsymbol{Y}$ : Producción de arroz $(\mathrm{kg} / \mathrm{ha})$.

Las variables regresoras son las que se muestran en la Tabla 1 anterior. Los resultados de la Tabla 4 fueron obtenidos mediante mínimos cuadrados ordinarios. Los errores estándar de los coeficientes son errores estándar robustos (New-West).

Las variables regresoras clave "compras estatales" y "precio estatal" pagado, fueron utilizadas con un rezago de un periodo para ambos modelos, debido a que según la teoría económica la adaptación de la oferta respecto a la demanda y las variaciones de los precios del mercado suele necesitar de un cierto plazo para verificarse, lo que da lugar a un retraso en la acomodación. El tiempo de cultivo que toma la producción de arroz es de cuatro a seis meses, en promedio, dependiendo de la variedad y condiciones de clima. Sin embargo, se tomó el rezago de un periodo con la finalidad de no perder grados de libertad y no debilitar las inferencias estadísticas que se desprenden de la estimación, dado que los resultados son muy similares (es decir también se utilizó más de un rezago, aunque con poca o igual significancia estadística de los resultados. También en ambos modelos se omitieron las variables precio en chacra pagado al productor, precio en la ciudad principal de cada región y el número de proveedores que atendieron la demanda estatal (como se dijo, a fin de evitar multicolinealidad).

En el caso de Lambayeque, en base a los resultados del modelo, se puede verificar diferencias entre los periodos de alta y baja producción de arroz. Los periodos de alta producción de arroz son mayo, junio y julio, los demás son de menor intensidad productiva. Según el coeficiente estimado (significativo), durante el trimestre mayo-julio se obtuvo una mayor producción de arroz, mayor en más de 4 mil Tm.

Por otro lado, en ambos modelos (Lambayeque y San Martín), la bondad de ajuste es bastante "aceptable", toda vez que los coeficientes de determinación $\left(\mathrm{R}^{2}\right)$ alcanzaron valores de $90 \%$ y $57 \%$, respectivamente. Se aprecia que en ambos casos solo podemos hacer inferencias respecto a la variable regresora "Área cosechada" ya que es la única variable que resultó estadísticamente significativa ( $\alpha=5 \%$ ), aún después de haber eliminado un problema original de multicolinealidad.

Destaca el hecho que ninguna de las variables "estatales" es estadísticamente significativa en ninguno de los dos modelos. Es decir que ni las "compras estatales" ni los "precios públicos" pagados por el arroz explican el comportamiento de la producción de arroz en los mercados regionales. Esto podría explicarse por el hecho que las compras estatales son relativamente "pequeñas" comparadas con el tamaño del mercado de arroz (nótese que las compras estatales escasa y eventualmente superan al $1 \%$ del total de arroz producido en cada región (Figs. 2 y 3 ).

En ese sentido, cabe realizar observaciones en ambas regiones sobre el comportamiento del mercado estatal durante el periodo de análisis, ya que según Federico Trionfetti (2000) los efectos que provoca la participación de las compras estatales están directamente ligados al tamaño del Estado (o a las entidades públicas) como demandante dentro del mercado.

\section{Participación de las adquisiciones estatales en la producción total de arroz}

En Lambayeque, a lo largo del periodo de análisis, utilizándose datos anuales, en casi todos los años, la participación de las compras de arroz realizadas por las entidades estatales oscila entre el $0,17 \%$ y el $0,55 \%$ de la producción total en dicha región. Solo en el año 2011 mostró un crecimiento importante llegando a 1,16\% (Fig. 2).

Tabla 4. Perú: Regresiones múltiples por región, producción-arroz como VD

\begin{tabular}{|c|c|c|c|c|}
\hline \multirow[b]{2}{*}{ Variables Regresoras } & \multicolumn{2}{|c|}{ LAMBAYEQUE } & \multicolumn{2}{|c|}{ SAN MARTIN } \\
\hline & Coeficiente & Err Est ${ }^{1}$. & Coeficiente & Err Est ${ }^{1}$. \\
\hline Intercepto & $-8.950,34 *$ & $4.162,78$ & $3.742,96$ & $3.380,83$ \\
\hline Tendencia & $-59,57$ & 37,89 & 27,09 & 50,36 \\
\hline Dummy $^{2}$ & $4.392,87^{*}$ & $2.021,00$ & -- & -- \\
\hline Compras estatales (-1) & $-4,45$ & 2,33 & $-0,17$ & 0,20 \\
\hline Precio estatal (-1) & 3,79 & 2,22 & $-2,66$ & 2,69 \\
\hline Área cosechada & $5,60 *$ & 0,11 & $4,75^{*}$ & 0,19 \\
\hline $\mathrm{R}^{2}$ & 0,90 & -- & 0,57 & -- \\
\hline Error Estándar de la Reg. & $4.158,61$ & -- & $7.491,54$ & -- \\
\hline
\end{tabular}

(*) Valor estadísticamente significativo con un margen de error del $5 \%$.

(1) Errores estándar robustos de Newey West.

(2) Variable ficticia que permite diferencias entre períodos de alta y baja producción de arroz.

Periodo: Enero 2006 - Diciembre 2011.

Muestra: Lambayeque, 36 observaciones. San Martín, 41 observaciones. 
Con algún grado de contraste, en San Martín también los porcentajes de compras de arroz a través del tiempo (respecto a la producción total) son relativamente bajos (2 $\%$ promedio en el período). Tal participación del Estado llegó a ser mayor; sin embargo, solo en los dos últimos años (6,4 \% durante el 2010 y 4,6 \% para el 2011). Durante los primeros cuatro años fue muy pequeña tal proporción, manteniéndose alrededor del 0,10\% del total producido (Fig. 3).

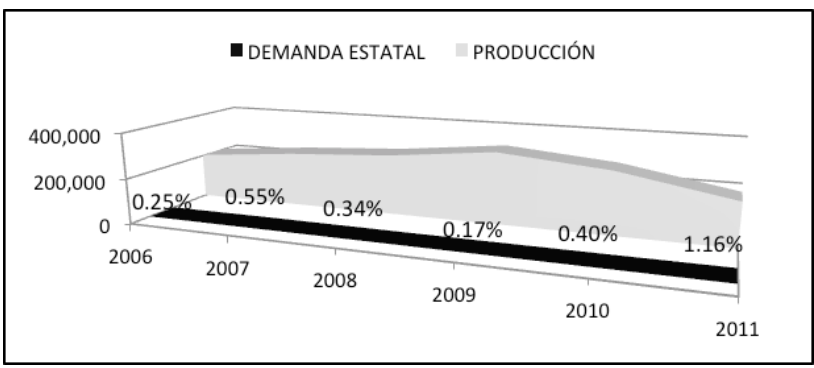

Figura 2. Perú: Participación en las compras estatales de arroz en Lambayeque (2006 - 2011)

Fuente: MINAG y SEACE (Perú).

\section{Comportamiento del mercado estatal de arroz en la región Lambayeque.}

Puede notarse también, de los resultados, que la estacionalidad de la producción es una característica clave en Lambayeque para el análisis del impacto de la demanda estatal sobre esta. Por un lado, las compras públicas no tuvieron un comportamiento compatible con la producción, es decir, no siempre se concentraron en los periodos de mayor producción de arroz (Fig. 4). Si

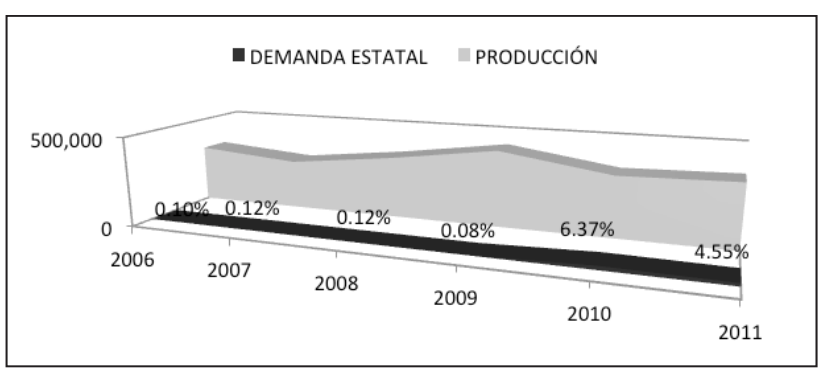

Figura 3. Perú: Participación de las compras estatales de arroz en San Martín (2006-2011)

Fuente: MINAG y SEACE (Perú).

bien el arroz es un producto que puede ser almacenado, no es rentable para los productores mantener un stock de producción, ya que deberán contar con capital para empezar con la nueva campaña de siembra.

En ese contexto, las compras públicas o estatales podrían ser más efectivas si se planificaran y se realizaran durante los periodos de mayor producción, a fin de absorber los excedentes de oferta de arroz. Otro aspecto importante son los plazos que se manejan para las adquisiciones, durante los procesos de selección y la ejecución de la compra en sí, con respecto a los plazos de entrega/pagos.

\section{Efecto de las compras y precios estatales sobre precios-} chacra

En este caso, los modelos pretenden explicar el comportamiento de los precios de arroz en chacra (soles por toneladas) en función de las variables independientes seleccionadas para cada una de las regiones incluidas (ver Tabla 2). También en este caso, la mejor forma funcional resultó la lineal, correspondiendo a la siguiente ecuación:

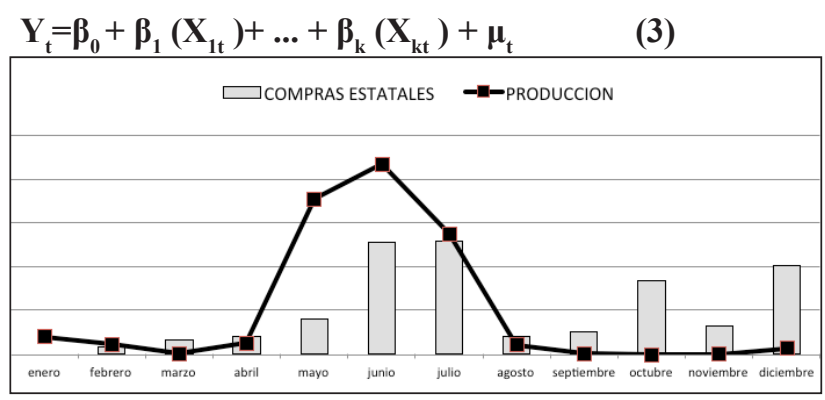

Figura 4. Perú: Estacionalidad de las compras estatales de arroz en Lambayeque (2006 - 2011)

Donde $\boldsymbol{Y}$ : Precio de arroz en chacra $(\mathrm{S} / \mathrm{Tm})$. Las variable regresoras son las que se muestran en la Tabla 2.

Las variables explicativas se tomaron con un rezago de un periodo para ambos modelos, la razón fue porque los efectos sobre los precios, teóricamente, podrían darse o producirse en "el siguiente periodo" por efectos de transmisión y ajuste de precios. En ambos modelos se omitieron -como variables regresoras- las variables "precio en la ciudad principal" de cada región, así como el número de proveedores que atendieron la demanda estatal y el área cosechada de arroz, evitando así la multicolinealidad.

Según datos de la Tabla 5, en ambos modelos finales la bondad de ajuste es relativamente alta (medida solo por los coeficientes de determinación). En el caso de Lambayeque el modelo explica el 59 \% de la variación de los precios en chacra; mientras que en el caso de San Martín, el modelo explica poco más del $57 \%$ de la variación del nivel de precios en chacra pagados al productor.

Se puede observar que, en este caso, ambas regiones muestran similares resultados. La variable "precio estatal" es estadísticamente relevante y la relación que muestra con la variable dependiente, precio en chacra del arroz, es positiva. De esa manera, en Lambayeque, por cada nuevo sol adicional que se paga por tonelada de arroz en el mercado estatal, en el siguiente mes el precio en chacra por tonelada de arroz se incrementaría en S/. 0,41; en San Martín sucede lo mismo en S/. 0,23 (Los valores promedio de precios estatales de los productos agrícolas, generalmente, son superiores a los precios promedio en el mercado privado, situación corroborada en el mercado estatal del arroz en Lambayeque y San Martín, ya que los precios estatales fueron superiores a los precios del arroz en la ciudad principal de cada región, durante todo el periodo de análisis). En el caso del modelo de San Martín, 
Tabla 5. Perú: Regresiones múltiples, precio en chacra como VD, por región

\begin{tabular}{|l|c|c|c|c|}
\hline & \multicolumn{2}{|c|}{ LAMBAYEQUE } & \multicolumn{2}{c|}{ SAN MARTIN } \\
\hline V. REGRESORAS & Coeficiente & Err Est. & Coeficiente & Err Est. \\
\hline Intercepto & 73,53 & 229,33 & $303,37^{*}$ & 121,61 \\
\hline Tendencia & 1,46 & 1,91 & $3,43^{*}$ & 1,56 \\
\hline Dummy & $-17,34$ & 93,65 & -- & -- \\
\hline Producción (-1) & $-0,001$ & 0,001 & $-0,003^{*}$ & 0,001 \\
\hline $\begin{array}{l}\text { Compras estatales } \\
(-1)\end{array}$ & 0,111 & 0,106 & 0,000 & 0,005 \\
\hline Precio estatal (-1) & $0,41^{*}$ & 0,12 & $0,23^{*}$ & 0,08 \\
\hline R2 & 0,59 & -- & 0,57 & -- \\
\hline $\begin{array}{l}\text { Error Estándar de la } \\
\text { Reg. }\end{array}$ & 183,49 & -- & $7.491,54$ & -- \\
\hline
\end{tabular}

${ }^{(*)}$ Valor estadísticamente significativo con un margen de error del $5 \%$.

(1) Errores estándar robustos de Newey West.

(2) Variable ficticia que permite diferencias entre períodos de alta y baja producción.

Periodo: Enero 2006 - Diciembre 2011.

Muestra: Lambayeque, 29 observaciones.

San Martín, 41 observaciones.

los resultados destacan las siguientes características adicionales: la tendencia que muestra la variable dependiente (precio de arroz en chacra) es significativa y positiva y existe relación negativa entre la producción de arroz y el precio en chacra, aunque de pequeña magnitud (0003), o sea cuanto mayor sea la oferta, en el siguiente mes los precios en chacra serían menores.

Este efecto estaría relacionado no solamente con las cantidades de arroz que se adquieren bajo modalidad pública, sino también con los precios que se traten dentro del mercado estatal del arroz. Ya que existen antecedentes que el Estado, a través de sus entidades públicas, realiza compras de productos agrícolas a precios superiores a los del mercado privado (Venero, 2012 y también Rebosio \& Rodriguez, 2001).

\section{Conclusiones}

Sobre la participación de los productores arroceros Entre el 2006 y el 2011, las asociaciones de pequeños agricultores fueron las que atendieron la mayor parte de la demanda estatal de arroz en Lambayeque y San Martín. Sin embargo en Lambayeque, las modalidades de adquisición de arroz ("subasta inversa") permitieron que las empresas comercializadoras intermediarias tuvieran también una importante participación en las provisiones (26 \%). En el caso de San Martín, con la modalidad de subasta convencional, fueron las asociaciones de productores las mayores participantes $(86 \%$ del total de provisiones estatales requeridas). Las condiciones y características que presentan los procesos de selección de proveedores para las adquisiciones de arroz dentro del mercado estatal, pueden ser consideradas un incentivo para la formación de asociaciones o empresas de productores, en ambas regiones. Estas conclusiones están basadas en datos primarios y formales del sector público; eventuales relaciones informales no han sido consideradas ni comprobadas (al no existir información para ello).

Sobre el efecto de las compras públicas en la producción de arroz

El tamaño del mercado estatal de arroz (compras de arroz por entidades públicas), respecto al mercado privado y total en ambas regiones, es muy pequeño para mostrar significativos efectos sobre la producción del mismo. Aunque la demanda de arroz por parte de entidades públicas muestra crecimiento en los últimos dos años del periodo de análisis (sobre todo para el caso de San Martín), las compras estatales siguen siendo relativamente mínimas y no han mostrado ser relevantes para explicar las variaciones en la producción total de arroz. Las compras públicas tuvieron un comportamiento temporal distinto o incompatible con la producción de arroz; es decir, las compras públicas no siempre se concentraron en los periodos de su mayor producción. Tal circunstancia es muy notoria en el caso de Lambayeque, un tanto menos en San Martín. Puede inferirse entonces que las compras públicas son poco efectivas pues no se realizan durante los periodos de mayor producción; es decir no "absorben" los excedentes de oferta de arroz (en ambas regiones). Entre las entidades que adquieren mayores volúmenes de arroz, destacan PRONAA, los gobiernos municipales y las Fuerzas Armadas, que demandan casi el $80 \%$ del total requerido por el sector público.

\section{Sobre el efecto en el precio en chacra}

Ambas regiones muestran similitudes en el efecto que tiene la variable "precio estatal" sobre el "precio en chacra". Ello denota que existiría un mecanismo importante de transmisión de precios del mercado estatal a los precios del arroz en chacra, que se muestra con mayor intensidad cuanto mayores sean los precios pagados por el Estado, respecto a los precios del mercado. El nivel o volumen de compras estatales no parece tener efecto significativo en los precios de arroz en chacra.

Además del efecto importante (relación directa) de la variable "precio estatal" como en la explicación del comportamiento de la variable "precio en chacra" (en ambas regiones), es importante añadir que la relación ha sido positiva y podría explicarse por la presencia histórica de las compras estatales (por más de 50 años) en ambas regiones. Todo ello a pesar de las cantidades relativamente pequeñas de las compras públicas, respecto al tamaño total del mercado de arroz en las regiones mencionadas (periodo 2006-2011).

Los precios "estatales" de los productos agrícolas han mostrado ser superiores a los precios en el mercado privado; situación que se pudo corroborar en el mercado de arroz de Lambayeque y San Martín. En alguna medida, ello también permite inferir que existiría la mencionada transmisión histórica de precios que finalmente ayuda a 
explicar la relación existente entre precios pagados por el sector público y los precios en chacra recibidos por los arroceros.

\section{Agradecimientos}

El estudio cuyos resultados aparecen en este artículo, fue apoyado y auspiciado por El Centro Peruano de Estudios Sociales (CEPES) y por el Proyecto "Mercados Campesinos". Los autores están muy agradecidos por ambos apoyos.

\section{Literatura citada}

Beláustegui, V. 2009. Las compras públicas sustentables en América Latina: Estado de avance y elementos clave para su desarrollo. Documento de Trabajo $\mathrm{N}^{\circ} 3$ del Programa ICT4GP. IDRC-OEA-USM.

Organización de las Naciones Unidas para la Agricultura y la Alimentación [FAO]. s/f. Disponible en http://faostat.fao.org.

Organización de las Naciones Unidas para la Agricultura y la Alimentación [FAO]. s/f. Revista AGRO-Noticias América Latina y el Caribe. UPI Noticias. Disponible en http://espanol.upi.com/ Economia/2013/06/25/.

Gonzales, D.; Jiménez, F. y Grippa, A. 2010. Informe de la cadena arroz - molinos. Lima- Perú: Ministerio de Agricultura.

Ley de Contrataciones del Estado 2009. Decreto Legislativo $\mathrm{N}^{\circ} 1017$.

Rebosio, G. y Rodríguez, E. 2001. Ingreso Campesino y Compras Estatales de Alimentos en el Perú. Lima, Perú: Atenea Impresores - Editores.

Rojas, G. y Flores, R. 2009. Estudio de mercado enfocado en priorizar las compras locales de alimentos a pequeños productores. Lima, Perú: Programa Mundial de Alimentos y Oficina de Naciones Unidas.

Rojas, M. y Effio, A. 2012. Modernización de las políticas de gestión de compras públicas en el Perú. Documento presentado en el XVII Congreso Internacional del CLAD: Reforma del Estado y de la Administración Pública. Cartagena, Colombia, 2012.

Sistema de Adquisiciones y Contrataciones del Estado [SEACE]. Disponible en http://www.seace.gob.pe.

Superintendencia Nacional de Administración Tributaria [SUNAT]. Disponible en http://www.sunat. gob.pe

Trionfetti, F. 2000. Discriminatory Public Procurement and International Trade. En The World Economy, pp. 5776.

Venero, J. 2012. Estudio de las Compras Públicas a los Pequeños Productores del Sector Agropecuario en el Perú. Mimeo. Lima, Perú.

Wooldridge, J. 2009. Introducción a la Econometría. (4ta. edición). México: Cengage Learning Editores. 